\title{
Elliptical dust growth in astrophysical plasmas
}

\author{
C. R. Stark, H. E. Potts, and D. A. Diver
}

Dept. of Physics and Astronomy, University of Glasgow, Glasgow G12 8QQ, Scotland, UK

e-mail: craig@astro.gla.ac.uk

Received 27 January 2006 / Accepted 27 June 2006

ABSTRACT

\begin{abstract}
Elongated dust grains exist in astrophysical plasmas. Anisotropic growth of elliptical dust grains, via plasma deposition, occurs if the deposited ions are non-inertial. In reality the extent of such growth depends upon the initial kinetic energy of the ions and the magnitude of the electric field in the sheath. Simulations of the dynamics of the ions in the sheath are reported, showing how elliptical growth is related to the initial eccentricity and size of the seed relative to the sheath length. Consequences for the eventual fate of elliptical dust are then discussed.
\end{abstract}

Key words. circumstellar matter - plasmas - dust, extinction

\section{Introduction}

Dust is ubiquitous in the universe. It exists in a variety of environments, such as the circumstellar dust shells of luminous post-main sequence stars where temperatures can be low enough $\left(T \sim 10^{2} \mathrm{~K}\right)$ to allow the growth of dust seeds via nucleation and condensation (Whittet 1992). The growth of dust grains precedes that of stellar, planetary, cometary and asteroid formation. The agglomeration of large dust grains into clumps leads to a rapid gravitational-like collapse, with a length much less than the Jeans length for gravitational collapse, aiding in the growth of these planetesimals (Bingham \& Tsytovich 2001). In such preplanetary systems dusty weakly ionised plasmas are responsible for the self-magnetisation of the environment and the matter within them (Wiechen 2004).

It is well known that the interstellar medium is populated by dust (Benoît et al. 2005; Leonard et al. 2002). Davis \& Greenstein (Davis \& Greenstein 1949) first proposed that the observed polarization of electromagnetic radiation from stars was the result of anisotropic extinction by aligned spheriodal grains in the interstellar medium. These grains collectively align themselves with the background galactic magnetic field via paramagnetic relaxation of the thermally rotating grains. However this mechanism assumes an abundance of paramagnetic material that is too large compared to the actual interstellar abundance. Grain alignment via a diamagnetic process removes this limitation (Chihara et al. 1998). Alternatively if you have a non-axisymmetric rotating charged grain in a magnetic field a magnetically induced torque realigns the grain (Diver \& Clarke 1996).

Supernova remnants are potentially a major source of interstellar dust in the Universe (Cernuschi et al. 1967; Hoyle \& Wickramasinghe 1970). The high abundance of heavy elements and the high density of the remnant appear to make the environment favourable for dust formation via various chemical and physical mechanisms. Recent observations of Cassiopeia A have revealed the presence of conducting metallic dust needles with a total mass of $10^{-4}$ to $10^{-3} M_{\odot}$ (Dwek 2004) opening a further diagnostic window on the environment via polarimetric studies of the polarization from the dust.
Much research has been carried out on the physical processes behind the formation of dust (Patzer et al. 1995; Nuth et al. 2002; Gordiets \& Bertan 2005) as well as on the effects spheroidal dust grains have on electromagnetic radiation (Gupta et al. 2005; Bazell \& Dwek 1990). This paper investigates the growth of spheroidal dust grains via plasma deposition and the applicability of this mechanism in supernova remnants.

Consider an ellipsoidal dust grain or seed present in a uniform electron-ion plasma. For a plasma in thermal equilibrium the greater mobility of the electrons ensures the grain is negatively charged resulting in a sheath region forming around the grain. There will now exist a flow of ions towards the grain which are deposited on the grain surface altering its size, mass, charge and hence the potential of the grain. This occurs until the grain reaches the floating potential $\phi_{\mathrm{f}}$ where the electron and ion currents are equal,

$\phi_{\mathrm{f}}=-\frac{k_{\mathrm{B}} T_{\mathrm{e}}}{2 e} \ln \left(\frac{m_{\mathrm{i}}}{2 \pi m_{\mathrm{e}}}\right)$

where $m_{\mathrm{i}}$ and $m_{\mathrm{e}}$ are the ion and electron mass respectively, $T_{\mathrm{e}}$ is the electron temperature and $k_{\mathrm{B}}$ is Boltzmann's constant. The sheath width is approximately a few electron Debye lengths $\lambda_{\text {De }}=\left(\epsilon_{0} k_{\mathrm{B}} T_{\mathrm{e}} / n_{\mathrm{e}} e^{2}\right)^{1 / 2}$, where the potential of the grain is shielded from the plasma noting that $n_{\mathrm{e}}$ is the electron density of the plasma (Lieberman \& Lichtenberg 2005). In this steadystate situation ions (plus their accompanying electrons) will be accelerated from the plasma via the presheath into the sheath region at the Bohm Speed, $u_{\mathrm{B}}=\left(k_{\mathrm{B}} T_{\mathrm{e}} / m_{\mathrm{i}}\right)^{1 / 2}$, where they will be deposited ultimately on the grain surface. When $\lambda_{\mathrm{De}}$ is much smaller than the ion mean free path, the Bohm criterion is necessary for the shielding by a stationary sheath (Riemann 1991).

For spherical dust grains the electric field is purely radial around the grain and deposits the ions uniformly on the grain surface. However ellipsoidal dust grains have a non-radial electric field close to the grain and ions could be deposited on the surface in a non-uniform fashion depending on their momentum, leading to growth in a preferred direction. If the momentum of the ions is too large they will not follow the direction of the field lines faithfully and resultant elongated growth may not occur. 
To see how the growth of elongated dust grains occur, the trajectories of the ions entering the sheath were studied. By considering a uniform distribution of ions at the sheath-plasma interface, each with a velocity equal to the Bohm speed, $u_{\mathrm{B}}$, the ion trajectories were calculated within the sheath. From these numerical calculations the points on the perimeter of the ellipse where the ions were deposited were calculated.

This paper addresses the problem of elliptical dust growth in electron-ion plasmas. In Sect. 2 a theoretical model of elliptical grain growth is discussed. Section 3 formulates the numerical algorithm used to calculate the electric field in the sheath surrounding the grains and hence the trajectories of the ions in the sheath. In Sect. 4 the results of the numerical simulations are discussed to see if elliptical growth occurs.

\section{2D Model: elliptical co-ordinates}

To simplify matters the problem will be restricted to $2 \mathrm{D}$ so that the sheath is a circle at the centre of which is an elliptical grain. It is assumed that the semi-major axis of the central elliptical crosssection is small compared to the sheath length in the plasma and that the grain is conducting. It is further assumed that the space between the grain and the sheath-presheath interface is a vacuum, in that there is no distribution of electrons or ions.

The electric potential of the dust grain can be expressed in elliptical co-ordinates as follows. The elliptical cross-section of the grain has cartesian co-ordinates given in parametric form as $(x, y)=(a \cos \theta, b \sin \theta)$, where $\theta$ is the angle subtended at the ellipse centre and $a$ and $b$ are the semi-major and minors axes of the ellipse respectively. The family of confocal ellipses that describe the contours of the electric potential are given by,

$\frac{x^{2}}{\xi^{2}}+\frac{y^{2}}{\xi^{2}-1}=1$

where $\xi>1$ and noting that the eccentricity, $e$, of the ellipse is given by $e=1 / \xi$. The electric field is expressed in the orthogonal co-ordinate system of confocal parabolas, given by

$\frac{x^{2}}{\eta^{2}}-\frac{y^{2}}{1-\eta^{2}}=1$

where $\eta<1$. In elliptical co-ordinates $\eta=\cos \theta$ where $\theta$ is the angle subtended by the asymptote to the parabola generated by the choice of $\eta$ (Abramowitz \& Stegun 1964). The coordinates are shown in Fig. 1.

\subsection{Anisotropic mass deposition}

Consider a uniform circular distribution of ions (and their accompanying electrons) moving into the sheath from the presheath and heading for deposition on the surface of the elliptical grain. The local thickness of the deposited layer depends strongly on the structure of the local electric field. In the case of a circular grain the distribution of ions would be deposited uniformly over the surface of the grain if they were to follow the purely radial electric field lines.

In the case of the elliptical grain, the electric field close to the grain surface is distorted from the purely radial electric field apparent at large distances. This distortion can be quantified by considering the intersection of the parabola that has asymptotes $\theta= \pm \pi / 4$ for which $\eta=1 / \sqrt{2}$. This parabola then intersects a quadrant of the ring of ions at the plasma, and if these ion trajectories were to follow exactly the electric field lines, then

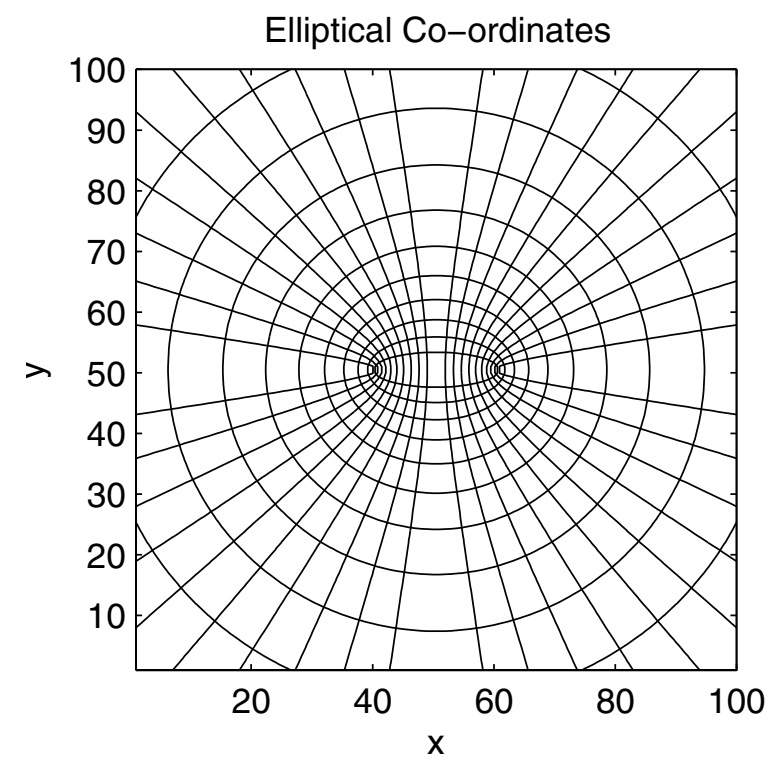

Fig. 1. Elliptical Co-ordinate system consisting of a system of confocal ellipses and parabolas which represents the electric potential and field respectively.

this material would be deposited on the section of the elliptic grain perimeter that is intersected by the parabola.

For a specific ellipse and parabola described by $\xi$ and $\eta$ respectively, Eqs. (2) and (3) can be solved to obtain the points of intersection of the ellipse and the parabola,

$x= \pm \eta \xi, \quad y= \pm\left[\left(\xi^{2}-1\right)\left(1-\eta^{2}\right)\right]^{1 / 2}$.

For the case of the parabola $\eta=1 / \sqrt{2}$,

$x^{*}= \pm \frac{\xi}{\sqrt{2}}, \quad y^{*}= \pm \frac{1}{\sqrt{2}}\left(\xi^{2}-1\right)^{1 / 2}$.

The parabola $\eta=1 / \sqrt{2}$ intersects the circle of matter at the plasma interface at the angles $\pm \pi / 4$. If all the charged material were to follow the parabolic electric field lines characterised by $1 / \sqrt{2} \leq \eta<1$ then this quadrant of the circular distribution of material would not intersect one quadrant of the elliptic surface, since the parabola subtends an angle $\theta^{*}$ at the ellipse centre, given by

$\tan \theta^{*}=\sqrt{\frac{\xi^{2}-1}{\xi^{2}}}=1-e^{2}$

which is only $\pm \pi / 4$ if $\xi=1 / e \gg 1$, that is, if $e \simeq 0$.

The arc length around an ellipse is given by the Incomplete Elliptic Integral of the second kind, denoted $E(\phi, m)$ where $\phi$ is the angle subtended at the centre of the ellipse by the arc, and $m=e^{2}$ is the elliptic parameter (Abramowitz \& Stegun 1964).

Consequently the ratio of the two arc lengths in the first quadrant created by the intersection of the ellipse and the parabola $\eta=1 / \sqrt{2}$ (see Fig. 2a) can be written as

$R^{*}=\frac{E\left(\pi / 2, e^{2}\right)-E\left(\theta^{*}, e^{2}\right)}{E\left(\theta^{*}, e^{2}\right)}$.

This function is graphed in Fig. 3, showing $R^{*}$ as a function of $e$. As $e \rightarrow 1$, the $\pi / 4$ segment of the circle at $\infty$ is mapped to an increasingly smaller fraction of the ellipse surface and $R^{*}>1$. 


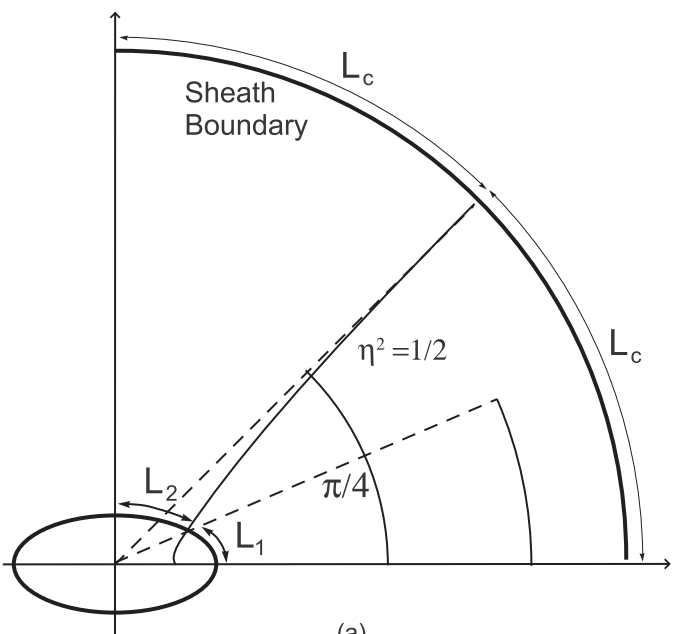

(a)

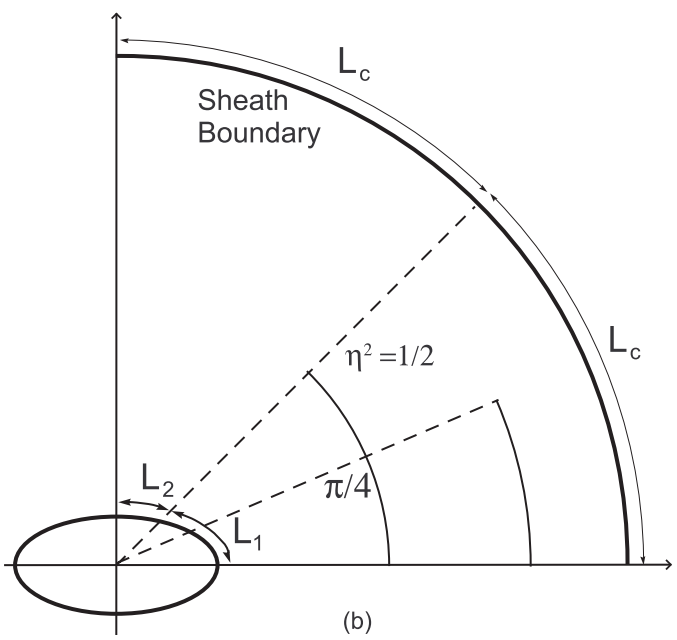

(b)

Fig. 2. Diagram a) shows the scenario when the ion follows the field lines faithfully giving the results plotted in Fig. 3 where $R^{*}=L_{2} / L_{1}$. Diagram b) shows the scenario when the ion at $\pi / 4$ travels undeviated towards the grain surface giving the results plotted in Fig. 5 where $R=$ $L_{2} / L_{1}$.

As $e \rightarrow 0$ material at $\infty$ is uniformly deposited over the surface since $E\left(\pi / 2, e^{2}\right) \rightarrow 2 E\left(\theta^{*}, e^{2}\right)$ hence $R^{*} \rightarrow 1$.

Assuming that charged particle trajectories follow the electric field, the amount of matter deposited on the elliptical surface for $1 / \sqrt{2} \leq \eta<1$ is identical to that deposited for $0 \leq \eta<1 / \sqrt{2}$, since the critical parabola $\eta=1 / \sqrt{2}$ bisects the quadrant at $\infty$. However, since $E\left(\pi / 2, e^{2}\right)>2 E\left(\theta^{*}, e^{2}\right)$ the mass gain of the polar region of the grain is greater than that of the equatorial regions.

\section{Numerical simulations}

A number of electric potentials were calculated with a range of grain scale size, $s$, and ratio of semi-major to minor axes, $a: b$. The scale size, $s$, of an ellipse of semi-major axis, $a$, is defined as $s=a / l$, where $l$ is the sheath length. Using the deposition sites of the ions on the surface of the ellipse, a measure of the grains elliptical growth was calculated,

$R=\frac{E\left(\pi / 2, e^{2}\right)-E\left(\theta^{\prime}, e^{2}\right)}{E\left(\theta^{\prime}, e^{2}\right)}$

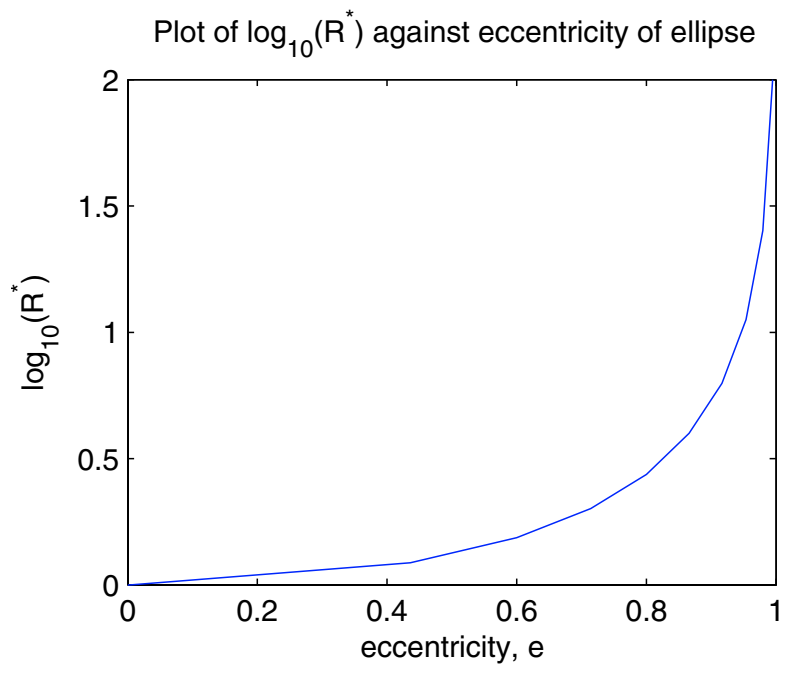

Fig. 3. Above graph shows that as $e \rightarrow 0, R^{*} \rightarrow 1$. As $e \rightarrow 1$ ellipse becomes more extreme and $R^{*} \rightarrow \infty$.

where $\theta^{\prime}$ is the angle subtended at the centre of the ellipse by the deposited ion that initially subtended an angle of $\pi / 4$ at the sheath boundary. For anisotropic mass loading leading to elliptical growth $R>1$. Note that $\theta \neq \theta^{*}$ since inertial particles don't follow the field lines.

\subsection{Electric potential of elliptical grain}

In order to obtain the electric potential of an elliptical grain Poisson's equation in two dimensions was solved numerically,

$\left(\partial_{x}^{2}+\partial_{y}^{2}\right) \Phi=0$

where $\partial_{x}\left(\partial_{y}\right)$ is shorthand for $\partial / \partial x(\partial / \partial y)$. Grain charging studies (such as Kersten et al. 2004) assume spherical, nonconducting grains preformed from insulating material. In the model presented here we are primarily concerned with grain growth over larger timescales in which inhomogeneous charge deposition is assumed to be averaged out, leaving only the gross geometrical effects controlling the total electrostatic field. Hence we will take the grain boundary to be an equipotential surface (but we note that random perturbations of charge deposition may well be a contributory factor that might merit investigation in a further study). The boundary and initial conditions are therefore,

$\Phi(x, y)= \begin{cases}0 & x^{2}+y^{2} \rightarrow \infty \\ \Phi_{0} & b^{2} x^{2}+a^{2} y^{2}-(a b)^{2} \leq 0\end{cases}$

in which $a$ and $b$ are the semi-major and minor axes of the elliptical grain respectively and $\Phi_{0}$ is the potential on the grain. To ensure that the boundary conditions were applied to the elliptical boundary and not a step-wise approximation to it an accurate field interpolator was used, the explanation of which follows.

The electric potential, $\Phi$, of a source in a vacuum decreases as $\propto 1 / R$, where $R$ is the perpendicular distance from the source. The perpendicular distance from the ellipse boundary to a mesh point $\left(x_{p}, y_{p}\right)$ is found by finding the critical points of the Lagrangian function

$L(x, y)=f(x, y)+\lambda g(x, y)$

where,

$f(x, y)=\left[\left(x_{p}-x\right)^{2}+\left(y_{p}-y\right)^{2}\right]^{1 / 2}$ 
$g(x, y)=\frac{x^{2}}{a^{2}}+\frac{y^{2}}{b^{2}}-1$

where $\lambda$ is the Lagrangian multiplier. Solving this yields a 4th order polynomial, solved numerically via a Newton-Raphson Method. Using this the perpendicular distance to the immediate mesh points outside the ellipse boundary was determined and the corresponding electric potential at these points calculated using,

$\Phi(x, y)=\frac{\gamma \Phi_{0}}{\gamma+R(x, y)}$

where $R(x, y)$ is the perpendicular distance from the ellipse perimeter to the mesh point $(x, y)$, and $\gamma$ is a normalization parameter.

\subsection{Ion trajectories in sheath region}

A fourth order Runge-Kutta numerical method was employed to calculate the trajectories of ions in the sheath. The model equations to be solved were:

$\left(\dot{u}_{x}, \dot{u}_{y}\right)=-\alpha\left(\partial_{x}, \partial_{y}\right) \Phi$

$(\dot{x}, \dot{y})=\left(u_{x}, u_{y}\right)$

where $\alpha=q_{\mathrm{i}} \phi_{0} / m_{\mathrm{i}} u_{0}^{2}$ is a dimensionless parameter in which $u_{0}$ is the launch speed of the ion and $\phi_{0}$ is the characteristic value of the electric potential which is taken to be the floating potential, $\phi_{0}=\phi_{\mathrm{f}}$. By altering $\alpha$ the ratio of the potential energy to the initial kinetic energy of the incoming ion can be changed. The circular distribution of ions at the sheath-plasma interface were initially given a radial launch velocity equal to the Bohm speed, $u_{0}=u_{\mathrm{B}}$; however, note that the parameter $\alpha$ is not constrained by the choice of $u_{0}$ or $\phi_{0}$.

\section{Results and discussion}

The variation of $R$ with $\alpha$ for elliptical grains of varying $a: b$ and differing scale sizes, s, are shown in Fig. 4.

When $\alpha \ll 1$ the electric potential energy of the ion is very small in comparison to its initial kinetic energy. In this case the influence of the potential on the ion is minimal and the ion's velocity will not be altered significantly, therefore $\theta^{\prime} \rightarrow \pi / 4$, see Fig. 2b. Due to the geometry of the ellipse it follows that $E\left(\pi / 2, e^{2}\right)-E\left(\pi / 4, e^{2}\right)<E\left(\pi / 4, e^{2}\right)$ hence,

$R \rightarrow \frac{E\left(\pi / 2, e^{2}\right)-E\left(\pi / 4, e^{2}\right)}{E\left(\pi / 4, e^{2}\right)}<1$

implying that the mass gain of the equatorial region is greater than that of the polar region and elliptical growth doesn't occur. This is evident in Fig. 4. The value of $R$ in this situation depends on the eccentricity, $e$, and decreases in value with increasing $e$, this is graphed in Fig. 5.

When $\alpha \gg 1$ the electric potential energy of the ion is now very large compared to its initial kinetic energy. The influence of the potential is greatly increased and will have a greater effect on the ions velocity, hence $\theta^{\prime} \rightarrow \theta^{*}$. Again due to the geometry $E\left(\pi / 2, e^{2}\right)-E\left(\theta^{*}, e^{2}\right)>E\left(\theta^{*}, e^{2}\right)$ therefore,

$R \rightarrow \frac{E\left(\pi / 2, e^{2}\right)-E\left(\theta^{*}, e^{2}\right)}{E\left(\theta^{*}, e^{2}\right)}>1$.
Now the mass gain of the polar region is greater than that of the equatorial and elliptical growth occurs. Assuming the ions follow the field lines exactly, it is expected in this case that since $\alpha \gg 1$ and $\theta^{\prime} \rightarrow \theta^{*}$ that $R \rightarrow R^{*}$, as graphed in Fig. 3, however as Figs. $4 \mathrm{a}-\mathrm{c}$ show this is not the case. Instead $R$ tends to some value, which is dependent on the eccentricity of the ellipse, that is much smaller than if the ions had followed the electric field lines faithfully. As $\alpha$ is increased and the ions respond to the potential more, there comes a point where the increase in the magnitude of the potential accelerates the ions to such high speeds that the potential close to the grain is not strong enough to alter their trajectories further. Therefore the value of $R$ saturates at some value.

Figure 4a, shows that elliptical grains of ratio $a: b=2: 1$ do not grow elliptically, implying that there is some value of eccentricity below which elongated growth doesn't occur. As $e \rightarrow 0$ the field close to the elliptical boundary becomes more radial and tends to that of a field for a circular grain. The field near the grain is therefore not strong enough, nor does it deviate from a radial field significantly to alter notably the trajectories of the ions close to the grain surface.

When $\alpha=1$ the electrostatic potential energy and the initial kinetic energy of the ions are equal. This signifies a point where the dynamics of the ions in the sheath change from being dictated by the electric potential of the grain to the initial kinetic energy of the ions.

Figure 6 shows $R$ against the scale size, $s$, when $\alpha=10^{6}$ for a number of eccentricities. The graphs show that for each eccentricity there exists a value of $s$ where $R$ is a maximum implying that there exists a size of grain that has optimum elliptical growth. If the scale size of the dust grain is very small compared to the sheath scale length, the ions will be accelerated by a nearly radial electric field for a large time and will tend to resist the influence of the non-radial field close to the surface of the grain due to its inertia. If the scale size of the dust grain is large compared to the sheath length, then the grain will appear planar at all points. Therefore the growth rate will be similar at all points on the surface.

Figure 6 and 4 also imply dust grains, in principle, will grow and pass through a series of evolutionary stages dependant on the eccentricity and scale size of the grain. Consider a dust grain of particular eccentricity, $e$, and scale size, $s$. For a certain value of $\alpha$ the grain will have a corresponding value of $R$ and a thin layer of material will be deposited on the grain surface such that $a \rightarrow a+\delta a$ and $b \rightarrow b+\delta b$. The value of $\delta a / \delta b$ depends on $R$ such that as $R \rightarrow 1, \delta a / \delta b \rightarrow 1$ and as $R \rightarrow \infty, \delta a / \delta b \rightarrow \infty$. The grains eccentricity, scale size and value of $R$ will change accordingly, which will in turn alter the ratio of $\delta a / \delta b$ and hence the way in which material is deposited on the grain surface further changing the eccentricity and scale size of the grain. This process continues until ultimately circular grain growth occurs where $\delta a / \delta b \rightarrow 1$.

The value of $\alpha$ in the simulations together with the choice of $q_{\mathrm{i}}, m_{\mathrm{i}}, \phi_{0}$ and $u_{0}$ define the physical system to which the results apply. Setting $\phi_{0}=\phi_{\mathrm{f}}$ and $u_{0}=u_{\mathrm{B}}$ the parameter $\alpha$ becomes

$\alpha=\frac{Z_{\mathrm{i}}}{2} \ln \left(\frac{m_{\mathrm{i}}}{2 \pi m_{\mathrm{e}}}\right)$

where $Z_{\mathrm{i}}$ is the net number of positive charges on an ion. For $a: b=3: 1$ elliptical growth occurs for values of $\alpha$ corresponding to plasmas composed of elements no lighter than Lithium, for $a: b=4: 1$ there is no such constraint on the elemental composition of the bulk plasma to give elliptical growth. This is 


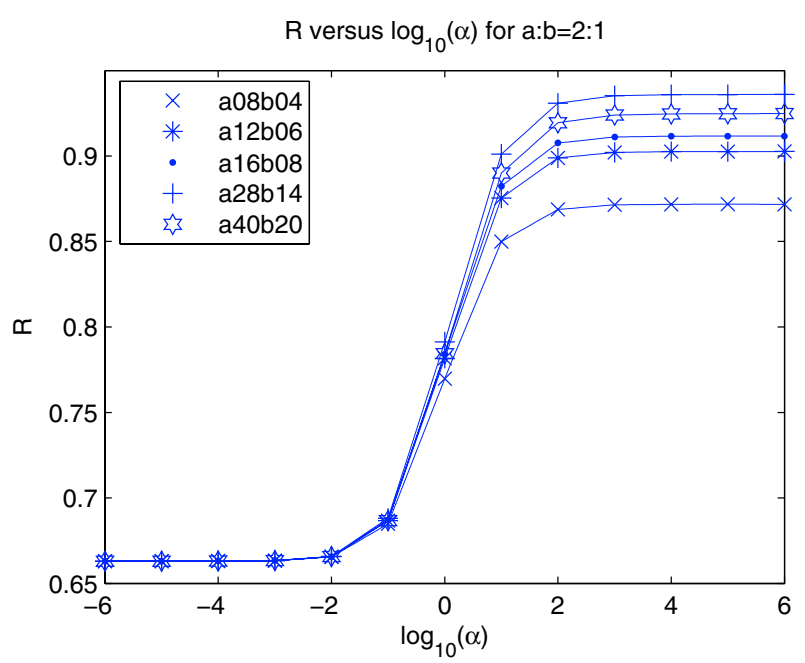

(a) $\mathrm{a}: \mathrm{b}=2: 1$

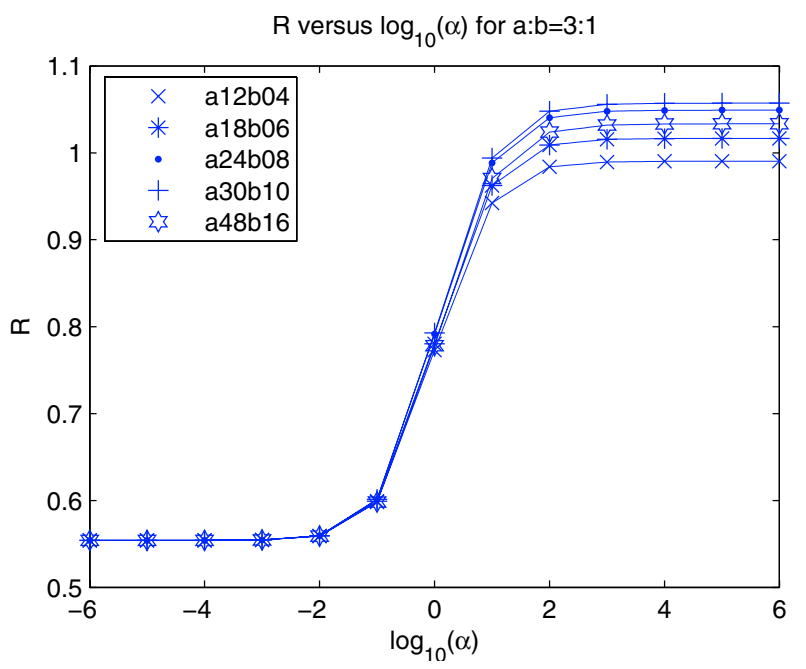

(b) $\mathrm{a}: \mathrm{b}=3: 1$

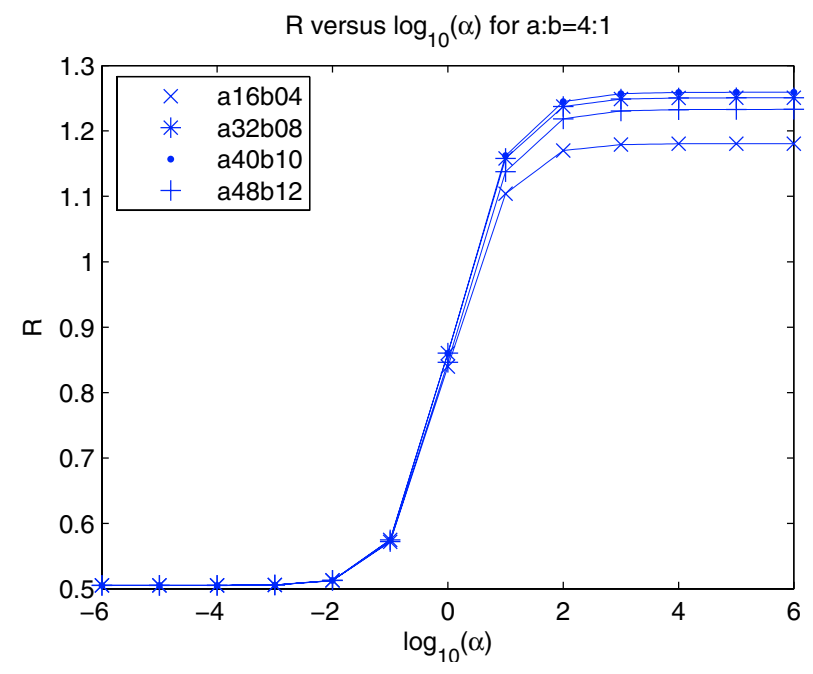

(c) $\mathrm{a}: \mathrm{b}=4: 1$

Fig. 4. $R$ against $\alpha$ for varying eccentricities, $e$, and for several scale sizes, $s$, where for example the notation $a 08 b 04$ represents an ellipse with a semi-major axis, $a$, of 8 units and semi-minor axis, $b$, of 4 units.

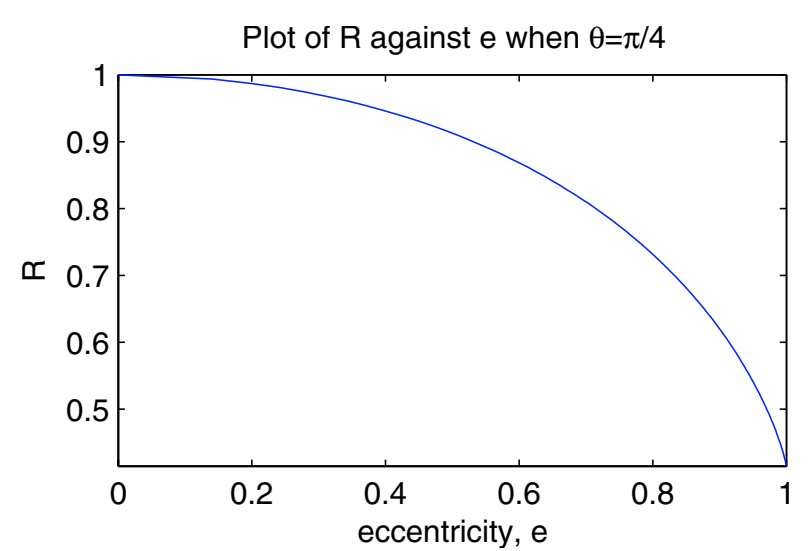

Fig. 5. As $e \rightarrow 1$ the ellipse becomes increasingly elongated and $R$ tends to a minimum.

providing the elements composing the plasma are fully ionised, however as heavier elements are considered elliptical growth of the grains is less constrained by the degree of ionisation of the plasma. Supernova remnants have very high abundances of heavy elements such as $\mathrm{O}, \mathrm{Si}$ and $\mathrm{Mg}$ suggesting the described growth mechanism may be a process present in remnants.

Given the potential on the grain surface, $\phi_{0}=\phi_{\mathrm{f}}$, if one assumes that the capacitance of the dust is that of a conductor of the same size then the net number of negative charges on the grain is given by

$$
N=\left(\frac{4 \pi \epsilon_{0} k_{\mathrm{B}}}{e^{2}}\right) T_{\mathrm{e}} \sqrt{a^{2}-b^{2}} \ln \left(\frac{m_{\mathrm{i}}}{2 \pi m_{\mathrm{e}}}\right) / \ln \left(\frac{a+\sqrt{a^{2}-b^{2}}}{a-\sqrt{a^{2}-b^{2}}}\right)
$$

For dust in laboratory and natural plasmas there typically exists a distribution of grain sizes $\sim 10^{-10}-10^{-6}$ metres and these can carry charges of up to $\sim 10^{6} \mathrm{e}$. Calculated values of $N$ yield $N \sim 10^{-1} T_{\mathrm{e}}$ for plasmas composed of elements lighter than Iron with grain sizes $\sim 10^{-6}$ where $a=3 b$ (note smaller grain sizes give $N<10^{-1} T_{\mathrm{e}}$ ). Supernova remnants can have an initial temperature of $\sim 10^{10} \mathrm{~K}$ which falls to lower temperatures as the remnant expands and cools. Elliptical growth as described here should occur in a supernova remnant given $T_{\mathrm{e}}$ is not too large that $N$ becomes inconsistently large. 


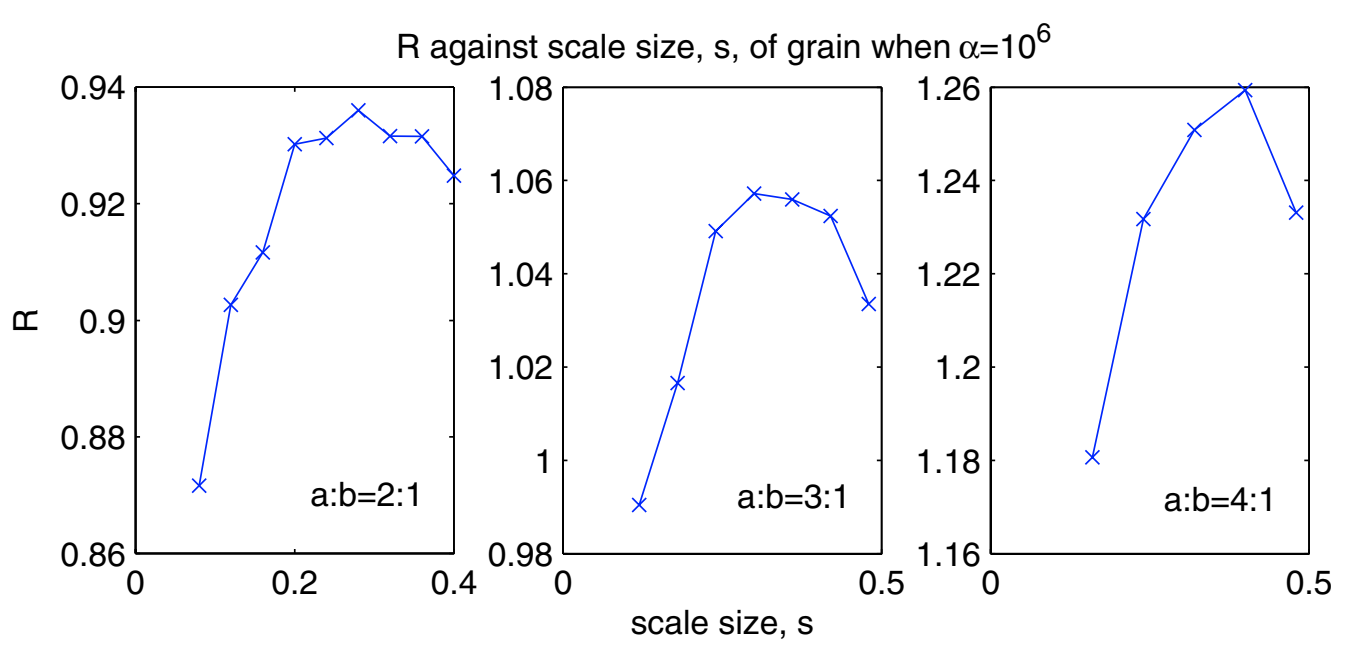

Fig. 6. The graphs show that for each eccentricity there exists a value of $s$ where $R$ is a maximum implying that there exists a size of grain that has optimum elliptical growth.

\section{Conclusions and further developments}

The numerical simulations have shown that within the model presented elliptical growth of dust grains occurs and that there exists an optimum scale size, $s$, that maximizes this elliptical growth. It was found however that only grains above a certain eccentricity displayed elongated growth when the potential energy of the ions in the sheath was much greater than their initial kinetic energy, $\alpha \gg 1$.

This suggests that if collisions between the ions and neutrals in the sheath are incorporated in the model elliptical growth may occur for lower values of $\alpha$. Collisions between ions and neutrals in a weakly ionised plasma would reduce the velocity of the ions and hence increasing $\alpha$ making them more susceptible to the field close to the grain.

The numerical simulations here assumed that the sheath region was a vacuum. However in reality, there exists a distribution of electrons and ions whose collective contribution to the field in the sheath may alter the nature of the grain's growth. The electron density in the sheath region would be described by the Boltzmann relation, assuming isothermal electrons, and would be $\propto \mathrm{e}^{e \Phi_{0} / k_{\mathrm{B}} T_{\mathrm{e}}}$. The ions assuming that $T_{\mathrm{i}}=0$ and that they enter the sheath at the Bohm speed, $u_{\mathrm{B}}$, have a density $\propto\left(1-e \Phi_{0} / 2 m_{\mathrm{i}} u_{\mathrm{B}}^{2}\right)^{-1 / 2}$ (Chen 1974). Due to the greater mobility of the electrons there will be a greater number of ions at all points in the sheath resulting in a positive contribution to the existing negative electric potential of the grain. This would alter the structure of the electric field in the sheath possibly encouraging elliptical growth.

The model adopted also excluded the presence of a background magnetic field, which can be instrumental in aligning the dust grains. Since elliptical dust grains grow in an environment where a field is present a more realistic model should incorporate a background magnetic field requiring the problem to be extended to 3 dimensions. In this context the formation of sheaths in the vicinity of a magnetic field would have to be considered; however the following simple argument shows how elongated grain growth may be encouraged when a magnetic field is included. In cylindrical polar coordinates consider a uniform magnetic field directed in the direction of the semi-major axis, $\boldsymbol{B}=B_{0} \hat{\boldsymbol{z}}$. The combination of the electric and magnetic field will produce an $\boldsymbol{E} \times \boldsymbol{B}$ drift in the $\hat{\boldsymbol{\theta}}$ direction, as well as particle gyrations around the magnetic field lines. Ions traveling parallel to the magnetic field will move unimpeded towards the grain while ions traveling perpendicular will now participate in orbits around the grain. Providing $|\boldsymbol{B}| \gg$ $|\boldsymbol{E}|$ ions will become trapped in these orbits stopping them from reaching the grain surface and thus encouraging growth at the poles. These developments are currently being addressed by the authors.

Acknowledgements. We are grateful for funding from PPARC via grant number PP/C000234/1 and for a studentship for C. R. Stark. We are grateful to the anonymous referee for positive comments and suggestions that have improved this paper.

\section{References}

Abramowitz, M., \& Stegun, I. A. 1964, Handbook of Mathematical Functions (New York: Dover Publications, Inc.)

Bazell, D., \& Dwek, E. 1990, ApJ, 360, 142

Benoît, A., Ade, P., Amblard, A., et al. 2005, A\&A, 424, 571

Bingham, R., \& Tsytovich, V. N. 2001, A\&A, 376, L43

Cernuschi, F., Marsicano, F., \& Codina, S. 1967, Ann. d'Ap., 30, 1039

Chen, F. F. 1974, Introduction to Plasma Physics and Controlled Fusion, Volume 1: Plasma Physics (KA/PP)

Chihara, H., Toshihide, O., \& Uyeda, C. 1998, J. Magnet. Mag. Mater., 177, 1455

Davis, L., \& Greenstein, J. L. 1949, Phys. Rev. Lett., 75, 10

Dwek, E. 2004, ApJ, 607, 848

Diver, D. A., \& Clarke, D. 1996, J. Phys. D: Appl. Phys., 29, 687

Gordiets, B. F., \& Bertan, E. 2005, Chem. Phys. Lett., 414, 423

Gupta, R., Mukai, T., Vaidya, D. B., Sen, A. K., \& Okada, Y. 2005, A\&A, 441, 555

Hoyle, F., \& Wickramasinghe, N. C. 1970, Nature, 226, 62

Kersten, H., Deutsch, H., \& Kroesen, G. M. W. 2004, Mass Spect., 233, 51

Leonard, D. C., Filippenko, A. V., Chornock, R., \& Weidong, L. 2002, ApJ, 124, 2506

Lieberman, M. A., \& Lichtenberg, A. J. 2005, Principles of Plasma Discharges and Materials Processing (Wiley-Interscience)

Morgan, H. L., Dunne, L., Eales, S. A., Ivison, R. J., \& Edmunds, M. G. 2003, ApJ, 597, L33

Nuth, J. A., Rietmeijer, F. J. M., \& Hill, H. G. M. 2002, Meteor. Planet. Sci., 37, 1579

Patzer, A. B. C., Köhler, T. M., \& Sedhnayr, E. 1995, Planet. Space Sci., 43, 1233

Riemann, K.-U. 1991, J. Phys. D: Appl. Phys., 24, 493

Wiechen, H. M. 2004, Icarus, 175, 15

Whittet, D. C. B. 1992, Dust in the Galactic Environment (IOP) 